\title{
Antroposofia aplicada à saúde em dez anos da Política Nacional de Práticas Integrativas e Complementares: aspectos históricos e considerações para sua implementação
}

Anthroposophy applied to Health in ten years of the National Policy of Integrative and Complementary Practices: historical aspects and considerations for its implementation Antroposofía aplicada a la salud en diez años de la Política Nacional de Prácticas Integradoras y Complementaria: aspectos históricos y consideraciones para su implementación

Iracema de Almeida Benevides ${ }^{1}$; Gisele Cazarin²; Sebastianjorge Flôrencio Ferreira de Lima ${ }^{3}$

\section{Resumo}

No Brasil, os campos da medicina antroposófica e da Antroposofia aplicada à Saúde vêm experimentando, nos últimos anos, significativa ampliação de sua representatividade no Sistema Único de Saúde, devido à sua crescente institucionalização no âmbito da Política de Práticas Integrativas de Saúde (PNPIC). Um dos eventos de maior destaque nessa trajetória foi a publicação, pelo Ministério da Saúde, da portaria GM 1.600/2006, que define as bases para a inserção dessa abordagem no SUS, sob forma de Observatório de experiências. O presente artigo apresenta os principais aspectos históricos desse processo, analisa seu desenvolvimento ao longo dos últimos dez anos e tece considerações sobre as perspectivas futuras considerando o cenário político-normativo do SUS.

Descritores: Antroposofia; Terapias Complementares; Sistema Único de Saúde.

\begin{abstract}
Anthroposophic Medicine and the whole field of Anthroposophy applied to Health in Brazil has been experiencing a significant increase in its representation in the scope of the Unified Health System in recent years thanks to its progressive institutionalization in the framework of the National Policy of Integrative and Complementary Health Practices (PNPIC). One of the most important events in this trajectory was the publication, by the Ministry of Health, of the normative 1.600 of July 17, 2006, which defines the bases for insertion of this approach in the SUS, in the form of Observatory of experiences. This article presents the main historical aspects of this process, analyses its development during the ten years of PNPIC publication and makes some considerations about the future perspectives considering the political and normative scenario of SUS.

Key-words: Anthroposophy; Complementary Therapies; Unified Health System.
\end{abstract}

\section{Resumen}

\footnotetext{
${ }^{1}$ Mestre Economia e Políticas de Saúde pela Universidade Bocconi de Milão, Itália. Afiliada à Associação Brasileira de Medicina Antroposófica (ABMA). Rua Tomé de Souza, 247, Funcionários, Belo Horizonte, MG. CEP: 30140-130. Email: iracema.benevides@gmail.com

2 Mestre em Saúde Pública pela Fundação Oswaldo Cruz/FIOCRUZ-PE. Docente e pesquisadora do Instituto de Medicina Integral Prof. Fernando Figueira (IMIP).

${ }^{3}$ Especialista em homeopatia pela Sociedade de Homeopatia de Pernambuco. Docente da Sociedade de Homeopatia de Pernambuco.
} 
ISSN 2179-6750

En Brasil, los campos de la medicina antroposófica y de la antroposofía aplicada a la salud vienen experimentando, en los últimos años, significativa ampliación de su representatividad en el Sistema Único de Salud, debido a su creciente institucionalización en el ámbito de la Política de Prácticas Integrativas de Salud (PNPIC). Uno de los eventos de mayor destaque en esta trayectoria fue la publicación por el Ministerio de Salud de la ordenanza GM 1.600 / 2006, que define las bases para la inserción de ese abordaje en el SUS, en forma de Observatorio de experiencias. El presente artículo presenta los principales aspectos históricos de este proceso, analiza su desarrollo a lo largo de los últimos diez años y toma consideraciones sobre las perspectivas futuras considerando el escenario político-normativo del SUS.

Palabras-claves: Antroposofía; Terapias Complementarias; Sistema Único de Salud.

\section{Introdução}

A Antroposofia aplicada à Saúde (AS) consiste num sistema médico-terapêutico complexo originado na Europa na época contemporânea ${ }^{1,2}$, que se caracteriza por uma abordagem ampliada dos problemas de saúde a partir de uma concepção integrada da relação do ser humano com a natureza, de seus aspectos biográficos, psicossociais e constitucionais, entre outros ${ }^{3}$. Essa abordagem integra o amplo panorama das Práticas Integrativas e Complementares em Saúde (PICS), expressão que corresponde, no Brasil, a Traditional Medicine/Complementar and Alternative Medicine (TM/CAM), nomenclatura utilizada pela Organização Mundial da Saúde $(\mathrm{OMS})^{4,5}$.

Na literatura internacional a expressão medicina antroposófica (MA) é utilizada de maneira ampla para denominar o sistema de cuidados, segundo a filosofia da Antroposofia, e que compreende a ampliação das práticas em saúde, a exemplo da medicina, enfermagem, odontologia, nutrição e psicologia, entre outros. Assim como, as práticas terapêuticas antroposóficas específicas como: a terapia artística antroposófica, massagem rítmica, euritmia, quirofonética, reorganização neuro funcional, aconselhamento biográfico, cantoterapia e musicoterapia antroposófica, entre outras. Uma das principais características da AS, desde o seu surgimento, é o modelo de atuação multiprofissional em que os diferentes profissionais de saúde envolvidos no cuidado buscam convergir e integrar os recursos terapêuticos, a partir de uma visão comum ${ }^{6}$.

No presente trabalho, o termo MA será utilizado prioritariamente para a denominação da prática específica dos profissionais médicos que ampliam sua atuação segundo os princípios dessa racionalidade, ao passo que a expressão AS compreenderá o conjunto da atuação dos diversos profissionais orientados segundo os referenciais da supracitada filosofia, e aplicados ao campo da saúde ${ }^{3,7}$.

No Brasil, a terminologia Práticas Integrativas e Complementares em Saúde (PICS) referese tanto aos sistemas médicos complexos, ou seja, às abordagens que preenchem as seis categorias 
ISSN 2179-6750

de racionalidades médicas propostas por $\mathrm{Luz}^{8}$, quanto aos recursos terapêuticos que podem estar associados a mais de um sistema de conhecimento, como é o caso das terapias manuais e do uso de plantas medicinais e fitoterápicos ${ }^{8,9}$.

Para Luz ${ }^{9}$, a crescente busca dos usuários pelas medicinas integrativas e complementares e sua progressiva incorporação nos sistemas locais de saúde está relacionada à baixa capacidade de resposta dos serviços de saúde no atendimento das necessidades dos usuários, fenômeno denominado por alguns autores de 'a crise da saúde'. Muitos usuários apontam que suas queixas não são ouvidas, que seus problemas reais não são abordados, e que sua saúde é fragmentada entre os diversos profissionais que atuam segundo o modelo convencional. Nesse sentido, as abordagens, ditas 'alternativas', são consideradas mais humanizadas, com o potencial de compreender melhor os sujeitos em sua totalidade existencial ${ }^{9}$.

Quanto aos paradigmas aplicados ao campo da saúde, $\operatorname{Luz}^{9}$ buscou definir os aspectos que compõem o referencial do modelo de medicina 'alternativa'. Esses aspectos dizem respeito à relevância da escuta do usuário, a partir da ampliação dos sentidos e da percepção pelo profissional de saúde, à centralidade do usuário no tratamento e a ênfase no cuidado e na abordagem terapêutica. Esse modelo se contrapõe paradigmaticamente ao modelo biomédico, que tem como eixo central a medicina convencional, acadêmica ou medicina mainstream ocidental, que surgiu da medicina hipocrática e passou por sucessivas mudanças paradigmáticas, acompanhando a evolução sóciohistórica ocidental. Configura-se como um modelo centrado na biologia humana e nas forças físicas da natureza, a partir do racionalismo europeu. No século XX, após as guerras mundiais, esse modelo desenvolve estreitas vinculações com a indústria farmacêutica e de tecnologias de diagnóstico, reforçando seu caráter de fragmentação e focalização na parte, em detrimento do todo $10-12$

No Brasil, a institucionalização dessas abordagens teve início na década de 1970 caracterizando-se pelo surgimento de experiências pontuais e isoladas. Reiteradas solicitações de usuários, gestores e profissionais de saúde em favor de sua implantação e seu fortalecimento no sistema público de saúde foram registradas em diversas Conferências de Saúde, desde a oitava edição da Conferência Nacional de Saúde (CNS), em 1986. Após a instituição do Sistema Único de Saúde (SUS) em 1988, e no contexto da crescente descentralização das ações de saúde, os estados e municípios conquistaram maior autonomia na definição de suas políticas locais. Isso criou ambiente político favorável a implantação de experiências pioneiras e inovadoras. No início dos anos 2000, com a publicação pela OMS do documento técnico Traditional Medicine Strategy 2002-2005 são lançadas as bases para a elaboração e 268 
ISSN 2179-6750

desenvolvimento de políticas públicas direcionadas às PICS no âmbito nacional ${ }^{3,11}$.

\section{Medicina antroposófica no contexto do Sistema Único de Saúde (SUS)}

A medicina antroposófica teve seu início, no Brasil, em 1956, na cidade de São Paulo (SP), com a atuação da médica generalista Gudrun Buckard, a qual havia realizado formação em uma das clínicas pioneiras na Europa. Os cursos para médicos, no país, abordando a prática clínica antroposófica tiveram início em 1976. No dos anos 1990 alcançaram ampliação com a instituição de cursos livres, porém com carga horária compatível com cursos de pós graduação lato sensu em diferentes regiões. A busca pelo reconhecimento dessa área de atuação, junto ao Conselho Federal de Medicina (CFM), foi iniciada ainda nos anos 1990, sem muita insistência frente aos pareceres alcançados ${ }^{3}$.

A homeopatia e a acupuntura trilharam um percurso diferenciado de institucionalização ao serem reconhecidas como especialidades médicas pelo Conselho Federal de Medicina na década de 1980. Em 1988, com a publicação das resoluções da Comissão Interministerial de Planejamento e Coordenação (Ciplan) foram fixadas as normas e diretrizes para o atendimento em homeopatia, acupuntura, termalismo, técnicas alternativas de saúde mental e fitoterapia. Cerca de uma década mais tarde (1999), os atendimentos realizados, por meio de tais práticas, foram incluídos na tabela de procedimentos do Sistema de Informação Ambulatorial (SIA/SUS), e passaram a ser remuneradas por procedimentos e monitoradas pelo volume de atendimentos ${ }^{13}$.

A trajetória da MA no âmbito social e comunitário teve seu início nos anos 1970, na favela Monte Azul em São Paulo (SP), com ações de atenção primária a saúde (APS) realizadas por profissionais com perfil generalista. $\mathrm{O}$ ambulatório médico terapêutico da Associação Comunitária Monte Azul contribuiu para a formação de muitos profissionais de saúde, especialmente médicos e enfermeiros. E, também, inspirou que posteriormente a MA fosse aplicada no SUS ou em experiências de medicina social e comunitária em algumas localidades das regiões sudeste e sul do país. A aproximação formal da MA com o SUS aconteceu em 1994, com a criação do programa de medicinas não convencionais da Secretaria Municipal de Saúde de Belo Horizonte. Na ocasião, essa abordagem foi incluída, juntamente com a homeopatia e a acupuntura/medicina tradicional chinesa (MTC), no Programa de Homeopatia, Acupuntura e Medicina Antroposófica (PRHOAMA).

Um evento decisivo no processo de institucionalização da MA foi a realização de concursos públicos para médicos antroposóficos no município supracitado, nos anos de 1994 e $1996{ }^{14}$. A oferta de serviços de MA tem se mantido estável no PRHOAMA, sendo desempenhada por quatro profissionais médicos, com atuação integrada à rede de atenção em saúde ${ }^{15-19}$. Outra experiência de 
ISSN 2179-6750

destaque da MA no âmbito da APS no SUS tem sido relatada em São João Del Rei, em Minas Gerais, desde $2002^{15,20}$.

\section{A construção da PNPIC e a integração da medicina antroposófica}

Em 2003, com o início dos trabalhos para a construção da Política Nacional de Práticas Integrativas e Complementares (PNPIC) no SUS, o grupo gestor responsável pela sua formulação, definiu a criação de subgrupos de trabalho específicos para cada uma das abordagens inicialmente definidas para integrá-la. Desse modo, a MA foi incluída juntamente com a fitoterapia, homeopatia e a MTC. A inserção da acupuntura deu-se devido à existência de experiências de institucionalização no SUS e na sociedade civil, além da importante defesa feita por técnicos no âmbito do Ministério da Saúde (MS) que conheciam essa abordagem 3,13,16,17.

O subgrupo de trabalho da MA realizou, em São Paulo (SP), em junho de 2004 um seminário para levantamento das propostas sobre a inserção dessa temática. Esse evento contou com a participação de técnicos do MS, estudantes, e profissionais de saúde do SUS. As propostas tiveram como foco o seguinte tema: 'Organizando a Assistência da Medicina Antroposófica no SUS'. A sistematização dos trabalhos foi entregue ao MS como contribuição para a implantação da MA na Política Nacional $3,13,16,17$.

O documento, a princípio intitulado de Política da Medicina Natural e Práticas Complementares (PMNPC), foi aprovado por unanimidade pelo Conselho Nacional de Saúde, após diversas instâncias de consultas e contribuições, vindo a ser, posteriormente, denominado de Política Nacional de Práticas Integrativas e Complementares (PNPIC), publicada por meio da Portaria $n^{\circ}$ 971/2006. Nesse contexto, a MA foi contemplada na PNPIC sob formato de Observatório das experiências de MA no SUS, após aprovação da Portaria n ${ }^{\circ} 1.600 / 2006{ }^{13}$, que posteriormente passou a ser considerada como parte integrante da PNPIC.

\section{Avanços na área da Assistência Farmacêutica relacionados às PICS e aos medicamentos antroposóficos}

Paralelamente à elaboração e aprovação da PNPIC aconteceram articulações para aprovação da Política Nacional de Plantas Medicinais e Fitoterápicos (PNPMF), concretizada por meio do Decreto $\mathrm{n}^{\circ}$ 5.813, de 22 junho 2006. A PNPMF, enquanto política interministerial, abrange toda cadeia produtiva e enfatiza o uso racional, a segurança, a eficácia e a qualidade desses recursos e insumos. Vinculada a essa política foram criados o Programa Nacional de Plantas Medicinais e Fitoterápicos e o Comitê Nacional de Plantas Medicinais e Fitoterápicos (Portaria Interministerial n ${ }^{\circ}$ 
ISSN 2179-6750

2.960/2008). Como fruto desse trabalho teve a elaboração da Relação Nacional de Plantas Medicinais de Interesse ao SUS, a RENISUS, composta por 71 espécies vegetais, das quais ao menos quinze são amplamente utilizadas na MA.

Uma importante conquista diretamente relacionada a MA consistiu na publicação da Resolução da Diretoria Colegiada (RDC) $n^{0}$ 26/2007, pela Agência Nacional de Vigilância Sanitária (ANVISA). Essa RDC dispõe sobre o registro de medicamentos dinamizados industrializados homeopáticos, antroposóficos e anti-homotóxicos. Esses últimos direcionados a facilitar o processo de eliminação, pelo organismo, de toxinas endógenas e exógenas ${ }^{21}$.

Igualmente relevante, nesse campo, foi a publicação da RDC n ${ }^{\circ}$ 67/2007 que regulamenta sobre Boas Práticas de Manipulação de Preparações Magistrais e Oficinais para Uso Humano em farmácias e seus Anexos. Tal norma define os requisitos mínimos exigidos para o exercício das atividades de: manipulação de preparações magistrais e oficinais das farmácias, desde suas instalações, aos equipamentos e profissionais, aquisição e controle da qualidade da matéria prima, armazenamento, avaliação farmacêutica da prescrição, manipulação, fracionamento, conservação, transporte, dispensação das preparações. Além da atenção farmacêutica aos usuários ou seus responsáveis, visando à garantia de sua qualidade, segurança, efetividade e promoção do seu uso seguro e racional.

\section{Antroposofia na Saúde no SUS após dez anos de PNPIC}

Entre os códigos que a Portaria n 85/2006 criou, o código 134-007 possibilita a identificação de serviços com PICS orientadas pela Antroposofia no Cadastro Nacional de Estabelecimentos de Saúde (CNES) ${ }^{16}$. Isso representou um avanço quanto ao registro da presença desse tipo de serviço e dos profissionais em atuação nos mesmos.

Entre as atividades do, anteriormente citado, Observatório, nos primeiros anos da PNPIC, a área responsável no Departamento de Atenção Básica (DAB/SAS/MS), em parceria com a Associação Brasileira de Medicina Antroposófica (ABMA) realizou o monitoramento dos principais serviços de MA nos anos de 2009 e 2010. Foi criado um questionário para ser enviado às experiências reportadas ao DAB, visando compensar a ausência de mecanismos formais de registro nos sistemas oficiais de informação. Dessa maneira, foram coletadas informações que permitiram conhecer as principais atividades realizadas pelos profissionais atuantes no SUS, além de experiências promovidas por 
ISSN 2179-6750

outras instituições vinculadas ou não ao SUS. Foram obtidas informações relativas aos números de atendimentos, os profissionais envolvidos, os insumos utilizados, assim como aspectos voltados à sua institucionalização e sustentabilidade ${ }^{22}$.

Das dezoito experiências analisadas, dezessete possuíam vinculação direta ou indireta ao SUS e o poder público. Apenas uma experiência não possuía vinculação, constituindo-se em um serviço independente (Ambulatório da Associação Comunitária Monte Azul (ACOMA). Ao todo, dez serviços estavam localizados em Minas Gerais (nos municípios de: Belo Horizonte, Betim, Oliveira, São João Del Rei, Juiz de Fora, Matias Barbosa e Santo Antônio do Amparo).

Constatou-se, por meio da mencionada pesquisa, que dez novos serviços de MA foram implantados no SUS após 2006, indicando que a publicação da PNPIC teve efeito indutor/impulsionador. Observou-se que cinco serviços funcionavam em parceria com o terceiro setor, quase sempre uma organização não governamental, como: Juiz de Fora, Matias Barbosa, São João Del Rei, Núcleo de Medicina Antroposófica da Universidade Federal de São Paulo (UNIFESP), e uma das experiências de Santo Antônio do Amparo. Essas experiências recebiam apoio financeiro de instituições antroposóficas viabilizando a contratação de médicos e terapeutas antroposóficos, a aquisição de insumos para as terapias e medicamentos e, em alguns casos, a realização de obras de adequação da unidade de saúde ${ }^{22}$.

Em outubro de 2016, o DAB/SAS/MS publicou informativo sobre a PNPIC, destacando informações colhidas durante o segundo ciclo de avaliação do Programa de Melhoria do Acesso e da Qualidade na Atenção Básica (PMAQ-AB), no ano de 2015. Do total de 5.666 equipes de Saúde da Família (eSF) que referiram realizar algum tipo de PICS, 242 relataram ações de AS. Em relação aos Núcleos de Apoio à Estratégia Saúde da Família (NASF), 16 do total de 393 núcleos estudados mencionaram trabalhar com PICS ${ }^{23,24}$.

No informativo de novembro de 2016, publicado pelo MS, foram apresentados dados do e-SUS referentes aos anos de 2015 (Figura 1) e parciais de 2016 (Figura 2). Em 2015, foram reportados 96.703 atendimentos em AS, de um total de 527.953 atendimentos individuais em 1.362 municípios distribuídos em 2.654 estabelecimentos de saúde ${ }^{23,24}$. 
Figura 1. Número de consultas por tipo de PICS, Brasil, 2015

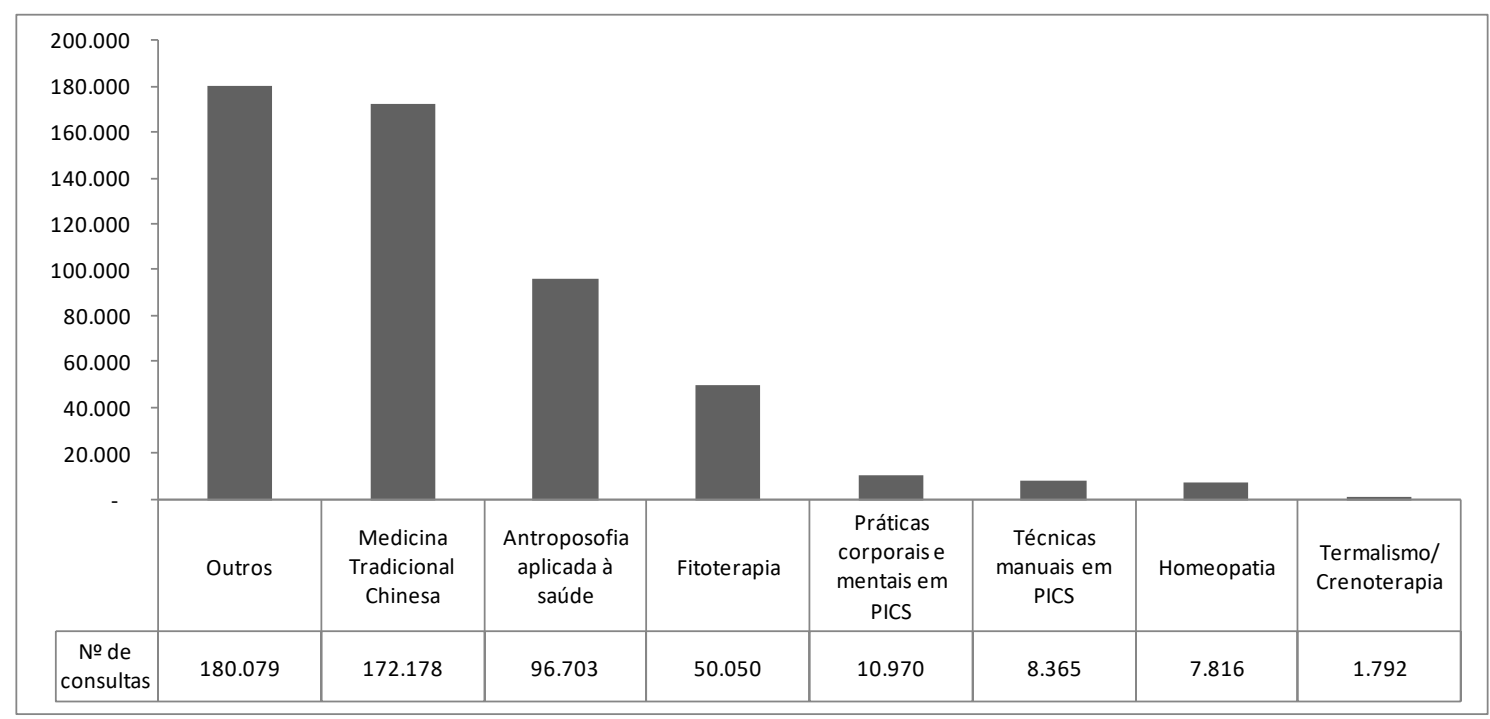

Fonte: e-SUS, DAB/SAS/MS (2015).

Figura 2. Número de atendimentos por tipo de PICS, Brasil, janeiro a agosto de 2016

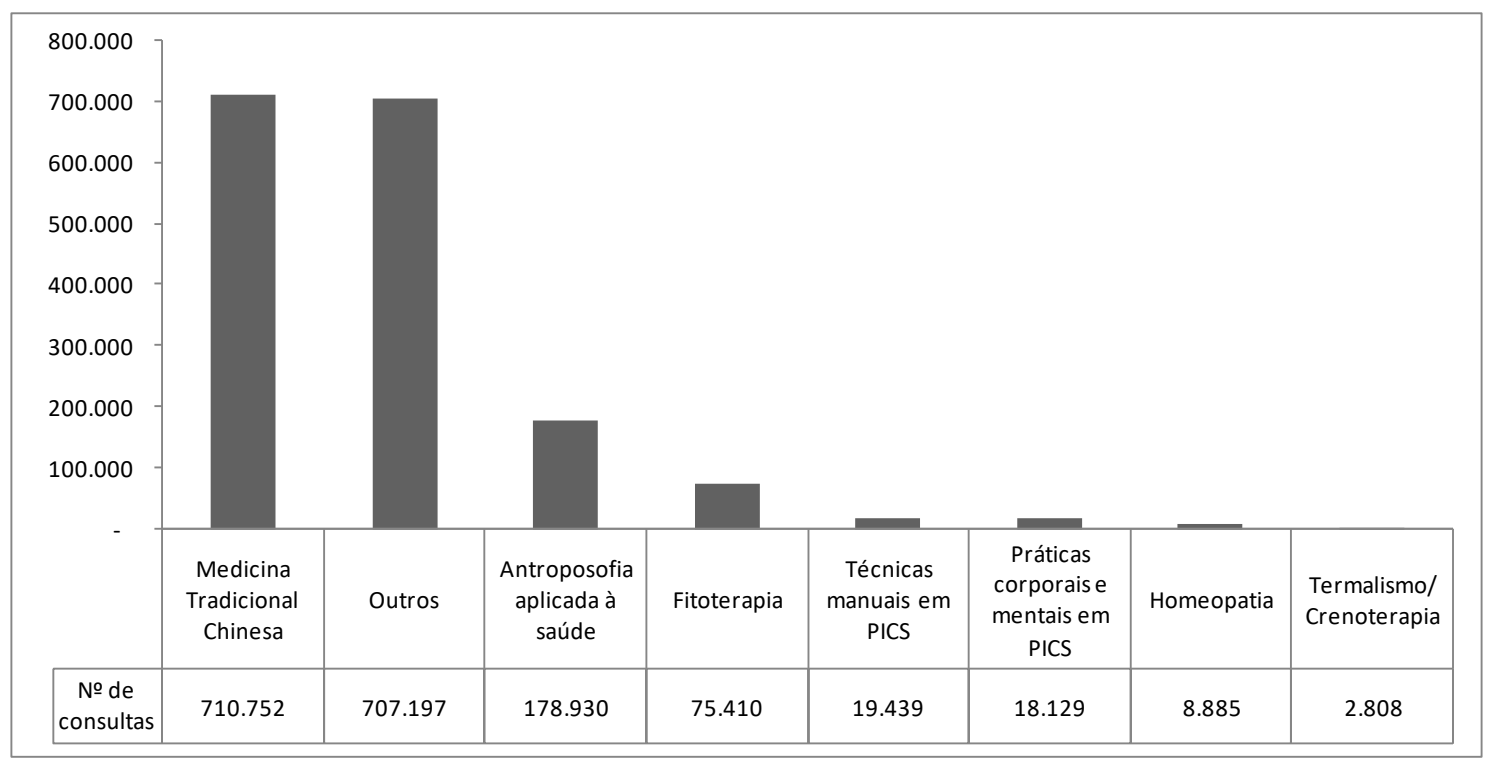

Fonte: e-SUS, DAB/SAS/MS (2016).

Em relação à ampliação da difusão dos conceitos e práticas da AS, um importante avanço foi possível devido ao fomento ao desenvolvimento e implantação do Curso de Introdução à Antroposofia na Saúde, na modalidade a distância, instalado em plataformas de acesso livre, vinculadas ao DAB/SAS/MS. Aproximadamente 2.000 indivíduos haviam sido alcançados por essa estratégia após oito meses de seu lançamento (Figura 3$)^{24}$. 
Figura 3. Número de participante por tipo de Curso EAD em PICS, Brasil, 2014-2016

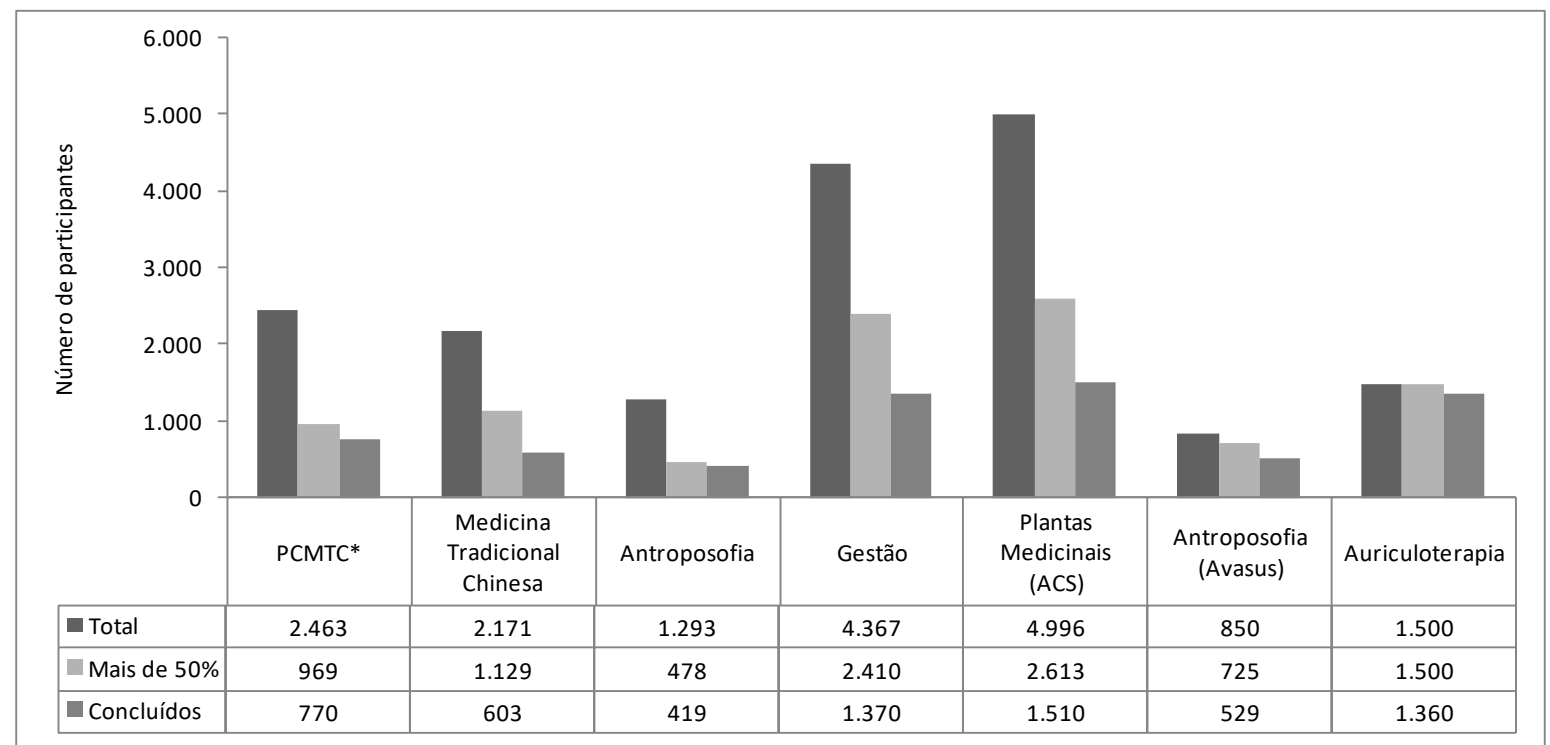

Fonte: DAB/MS (2016).

Ao longo de dez anos de PNPIC, experiências de AS ganharam espaço em universidades, desde apresentações pontuais em programas de graduação, até experiências mais consolidadas como a do Núcleo de Medicina Antroposófica (NUMA) e do Ambulatório de AS/Neurologia, ambos da Universidade de São Paulo (UNIFESP). Vale destacar que a Chamada MCTI/CNPq/MS - SCTIE Decit $n^{\circ}$ 07/2013 viabilizou a realização de quatro projetos relativos à AS, cujos resultados deverão ainda ser publicados.

\section{Perspectivas para ampliação da AS no SUS}

Embora as experiências de AS representem um contingente, ainda, limitado se comparadas a outras abordagens de PICS, avanços inegáveis têm acontecido na conquista de espaços para sua implementação no SUS. Assim como em outras PICS, deve ser ressaltado o grande mérito dos profissionais que fizeram essa escolha e perseveram cotidianamente pela manutenção dos espaços conquistados, vivenciando frequentemente limitações e contextos, muitas vezes desfavoráveis.

Em relação ao monitoramento, o CNES ainda não reflete a capilaridade dos serviços de AS, conforme orientação da Portaria $\mathrm{n}^{\circ}$ 853/2006. A implantação e aperfeiçoamento do e-SUS e a possibilidade de registro de atendimentos de AS apresenta-se como um importante marco para o monitoramento de indicadores nessa área. Entretanto, faz-se necessário que os sistemas de informação possam vir a registrar toda a ampla gama de abordagens que integram a AS. Paralelo ao monitoramento, a implantação de estratégias de avaliação possibilitará conhecer a realidade dos 274 
ISSN 2179-6750

serviços e suas as práticas.

A implementação dessa área certamente deverá passar pela ampliação e aperfeiçoamento, substituindo o estágio de Observatório de Medicina Antroposófica por um marco normativo que venha a contemplar a totalidade de práticas e abordagens profissionais compreendidas na AS.

Importante ressaltar também que expansão da MA e suas terapias deve ser acompanhada por soluções para a assistência farmacêutica, identificando os instrumentos que possibilitem a sua viabilização, seja por meio de aquisição dos industrializados pelos gestores, seja por meio da utilização dos homeopáticos e fitoterápicos como equivalentes, ou ainda, pela proposição de medicamentos antroposóficos manipulados.

\section{Referências}

1. Kienle GS, Albonico H-U, Baars E, Hamre HJ, Zimmermann P, Kiene H. Anthroposophic Medicine: an integrative medical system originating in Europe. Glob Adv Health Med. 2013 Nov;2(6):20-31. https://doi.org/10.7453/gahmj.2012.087.

2. Follador ECR. Medicina Antroposófica: um novo paradigma para as questões da medicina moderna. Rev Med. 2013 Set;92(3):166-72. http://dx.doi.org/10.11606/issn.1679-9836.v92i3p166-172.

3. Benevides I. Resultado do estudo de campo: a medicina e o médico antroposófico nas vozes de quatro gerações brasileiras distintas. In: Luz MT, Afonso VW, organizadores. Medicina antroposófica como racionalidade médica e prática integral de cuidado à saúde: estudo teórico-analítico e empírico. Juiz de Fora: UFJF; 2014. p. 103-64.

4. WHO global strategy on traditional and alternative medicine. Public Health Rep. 2002 May-Jun;117(3):300-1.

5. World Health Organization. Traditional Medicine Strategy: 2014-2023. Hong Kong: WHO: 2013. $76 \mathrm{p}$.

6. International Federation of Anthroposophic Medical Associations. The system of anthroposophic medicine. Brussels: IVAA; 2014. 56 p.

7. Luz MT, Wenceslau LD. A medicina antroposófica como racionalidade médica. In: Luz MT, Afonso VW, organizadores. Medicina antroposófica como racionalidade médica e prática integral de cuidado à saúde: estudo teórico-analítico e empírico. Juiz de Fora: UFJF; 2014. p. 73-101.

8. Luz MT. Medicina e racionalidades Médicas: estudo comparativo da medicina ocidental 275 
contemporânea, homeopática, tradicional chinesa e ayurvédica. In: Canesqui AM, organizador. Ciências sociais e saúde para o ensino médico. São Paulo: FAPESP; 2000. p. $181-200$.

9. Luz, MT. Cultura contemporânea e medicinas alternativas: novos paradigmas de saúde no fim do século XX. Physis. 2005;15(Supl):145-76. http://dx.doi.org/10.1590/S010373312005000300008 .

10. Barros, NF. A Construção de novos paradigmas na medicina: a medicina alternativa e a medicina complementar. In: Canesqui AM, organizador. Ciências sociais e saúde para o ensino médico. São Paulo: FAPESP; 2000. p. 201-13.

11. Barros, NF. A Construção da medicina integrativa: um desafio para o campo da saúde. São Paulo: Hucitec; 2008.

12. Ibañez, N, Marsiglia, R. Medicina e saúde: um enfoque histórico. In: Canesqui AM, organizador. Ciências sociais e saúde para o ensino médico. São Paulo: FAPESP; 2000. p. 49-73.

13. Brasil, Ministério da Saúde, Secretaria de Atenção à Saúde, Departamento de Atenção Básica. Política Nacional de Práticas Integrativas e Complementares no SUS - PNPICSUS. Brasília, DF: Ministério da Saúde, 2006. 92 p. (Série B; Textos básicos de saúde).

14. Cançado MRR, Gonçalves CG, Soares IA. Atendimentos em medicina antroposófica no SUS (Sistema Único de Saúde) em Belo Horizonte. Arte Med Ampl. 2000;20(3):31-6.

15. Revista Brasileira de Saúde da Família. Brasília, DF: Ministério da Saúde. Ano IX, Edição especial, 2008.

16. Benevides I. Inserção da medicina antroposófica no Sistema Único de Saúde: aspectos históricos, marcos normativos e desafios para sua implementação. Arte Med Ampl. 2012 jan-mar;32(1):4-11.

17. Benevides I. Observatório dos serviços de medicina antroposófica no Sistema Único de Saúde: resultados parciais do monitoramento no período 2009-2011. Arte Med Ampl. 2012;32(1):26-34.

18. Secretaria Municipal de Saúde de Belo Horizonte. PRHOAMA: programa de homeopatia, acupuntura e medicina antroposófica no SUS, BH. Belo Horizonte: Secretaria Municipal de Saúde; 2004.

19. Secretaria Municipal de Saúde de Belo Horizonte. PRHOAMA: programa de homeopatia, acupuntura e medicina antroposófica 21 anos: um verdadeiro encontro com a saúde. Belo Horizonte Secretaria Municipal de Saúde; 2015. 
20. São João del Rei. Lei Municipal no. 6.621, de 12 de novembro de 2013. Institui a Política Municipal de Práticas Integrativas e Complementares (PMPIC). Diário Oficial do Município de São João Del Rei; 2013 nov 12.

21. Scabello RT, Gardin, NE. Medicamentos dinamizados injetáveis disponíveis no Brasil: indicações baseadas na homotoxicologia e possibilidades de uso pela visão antroposófica. Arte Med Ampl. 2015 jul-set;35(3):118-25.

22. De Simone C, Antoni CD, Benevides I, Brina NT. Observatório dos serviços de medicina antroposófica no SUS resulatdos parciais do monitoramento no período 20092011. In: Luz MT, Afonso VW, organizadores. Medicina antroposófica como racionalidade médica e prática integral de cuidado à saúde: estudo teórico-analítico e empírico. Juiz de Fora: UFJF; 2014, p. 165-80.

23. Ministério da Saúde, Departamento de Atenção Básica. Informe política nacional de práticas integrativas e complementares. Brasília, DF: MS; 2016 outubro.

24. Ministério da Saúde, Departamento de Atenção Básica. Informe política nacional de práticas integrativas e complementares. Brasília, DF: MS: 2016 novembro. 